\title{
IMMUNOHISTOCHEMICAL AND ELECTRON MICROSCOPICAL STUDIES ON SEROTONIN-CONTAINING NERVE FIBERS IN THE PINEAL ORGAN OF THE RAT
}

\author{
TADAO MATSUURA, YOSHIHIRO TAKEUCHI and YUTAKA SANO \\ Department of Anatomy, Kyoto Prefectural University of Medicine, Kawaramachi Hirokoji, Kamikyoku, \\ Kyoto 602, Japan
}

\begin{abstract}
An investigation was made on the development of serotonin immunoreactivity in pinealocytes and nerve fibers in the pineal organ of rats, $0-21$ days after birth, using the peroxidase-antiperoxidase (PAP) immunohistochemical technique. Serotonin immunoreactivity in pinealocytes and nerve fibers could be detected earlier by the PAP method than by the formaldehyde-induced fluorescence histochemical method used by previous authors. Immunopositive cells appeared scattered in the gland already one day after birth. Serotonin-containing fibers with varicosities were found in the gland three days after birth. As days passed after birth, the serotonin immunoreactivity in the pinealocytes became stronger and intercellular elements also came to be immunostained, suggesting release of serotonin from the cells either physiologically or artifactually; the reaction in the nerve fibers became gradually localized in the varicose terminals. From the immunohistochemical findings and submicroscopical results after treatment with 5-hydroxydopamine and 5,6-dihydroxytryptamine, it is inferred that the noradrenergic pineal nerves take up serotonin released from the pinealocytes and gradually accumulate it in their varicose terminals as the rat matures. It is presumed to be stored both in the agranular and granular vesicles in a concentrated form.
\end{abstract}

Distribution of sympathetic postganglionic fibers in the pineal organ of various mammals has been investigated in detail by many researchers by means of formaldehyde-induced fluorescence histochemistry (FIFH). Through a series of these studies, it has been demonstrated that the noradrenergic nerve fibers distributed in the stroma of the pineal organ contain indolamine, viz., serotonin $(5,8,18-20,22,23,26)$. On the basis of studies on quantitative and qualitative changes in pineal monoamines after transplantation of the pineal organ into the anterior chamber, after treatment with a false transmitter $(\alpha-$ methyl-m-tyrosine), or after other surgical or chemical treatment, Bertler ef al. (5) and Owman (22) concluded that pineal noradrenergic fibers took up serotonin produced in the pinealocytes and stored it in their terminal portions. However, the mechanism of the uptake of serotonin into the axoplasm and the biological significance of these phenomena, and also the developmental quantitative changes in serotonin content in the pineal nerve fibers, have not yet been morphologically clarified.

In the present study the quantitative changes in serotonin content in the pineal organ, viz., in the pinealocytes and sympathetic nerve fibers, in the postnatal developmental stages of the rat were studied by a modified peroxidase-antiperoxidase (PAP) method using serotonin antiserum. In addition, the storage site of serotonin in the axoplasm of the pineal nerve fibers was electron-microscopically investigated using rats given false transmitters. 


\section{MATERIALS AND METHODS}

Wistar rats in various stages of development $(0$, $1,3,7,10,14$ and 21 days after birth) and adults (250-300 g body weight) of both sexes were used in this study. They were kept under conditions simulating equal hours of daylight and darkness at $18-22^{\circ} \mathrm{C}$, and were killed between 11:00 and 14:00 under sodium pentobarbital anesthesia. Animals at each stage of development, including adult rats, were divided into two groups, one for immunohistochemical study and the other for submicroscopical study. Bilateral superior cervical ganglionectomy was performed on four adult rats. Eight days after the operation they were sacrificed, two for immunohistochemical and two for electron-microscopical studies.

\section{Immunohistochenical Procedure}

Three rats at each stage of development, including adult rats, were used for immunohistochemical study. The animals were perfused with saline under anesthesia to wash out the blood, and then fixed by perfusion with a mixture of $0.3 \%$ glutaraldehyde, $4 \%$ paraformaldehyde, and $0.5 \%$ picric acid in $0.1 \mathrm{M}$ phosphate buffer at $\mathrm{pH} 7.4$ via the left cardiac ventricle. The pineal organ was postfixed with the same fixative without glutaraldehyde for two days at $4^{\circ} \mathrm{C}$ and then was immersed in $20 \%$ sucrose in phosphate buffer for two days at $4^{\circ} \mathrm{C}$. Frozen sections of $20 \mu \mathrm{m}$ thickness were prepared with a cryostat. Immunohistochemical staining was performed by the peroxidase-antiperoxidase (PAP) method on free-floating sections. Serotonin antiserum was used at 1:10,000 dilution. The antibody used in this study was raised in male rabbits against an antigen prepared by coupling serotonin to bovine thyroglobulin with a formaldehydeinduced reaction (for details, see reference 25). Immunoreactivity in the pineal organ was negative when the antiserum was absorbed by synthetic serotonin (50 $\mu \mathrm{M}$ serotonin in $1: 10,000$ antiserum) before application.

\section{Electron Microscopical Procedure}

Three rats at each developmental stage and three adult animals were used. In six adult rats a false transmitter, 5-hydroxydopamine (5-OHDA, $100 \mathrm{mg} / \mathrm{kg})$ or 5,6-dihydroxytryptamine $(5,6-$ DHT, $100 \mathrm{mg} / \mathrm{kg}$ ), was administered intraperitoneally $5 \mathrm{~h}$ before decapitation. Immediately after decapitation under anesthesia, the pineal organ was excised and immersed in $2.5 \%$ glutaraldehyde in $0.1 \mathrm{M}$ phosphate buffer at $\mathrm{pH}$ 7.4 for $2 \mathrm{~h}$ at $4{ }^{\circ} \mathrm{C}$, and then postfixed with $1 \%$ osmium tetroxide in the same buffer for $1 \mathrm{~h}$ at $4^{\circ} \mathrm{C}$. The specimens were dehydrated with a graded series of acetone and embedded in Lubeak 812 (Nakarai Chem. Co.). Ultrathin sections were stained with uranyl acetate and lead nitrate.

\section{RESULTS \\ Immunohistochemical Findings}

Adult Abundant distribution of serotonin immunoreactive fibers was observed in the pineal organ of adult animals. They were typical beaded fibers with numerous varicosities and formed a basket-like network in the perivascular space (Fig. 3a). A small number of serotonin fibers branching from the perivascular plexus penetrated into the intercellular space of the parenchyma as isolated fibers. The diameter of each varicosity was about $1.5 \mu \mathrm{m}$. Since the intervaricose segments of serotonin fibers were very thin and the immunoreactivity of these parts was weak, serotonin fibers were often seen as dark brown dots arranged in a line.

A dense distribution of varicose serotonin nerve fibers was observed in the area of the brain near the pineal organ, e.g., in the habenular nucleus and in the basal part of the subcommissural organ. Some of these fibers entered the pineal stalk, but it was not possible to obtain conclusive evidence that these serotonin fibers penetrated directly from the brain into the pineal organ through the pineal stalk (Fig. 2).

Eight days after the surgical removal of bilateral superior cervical ganglia, the immunoreactive fibers in the pineal organ completely disappeared, but no change was observed in the immunoreactivity of the parenchymal cells. We therefore conclude that all of the serotonin fibers in the pineal organ originate from the cervical sympathetic ganglia.

Since the pineal parenchyma, including the stroma, showed intense reactivity against the serotonin antiserum, it was difficult to identify individual cells (Figs. 1 and 3a).

\section{Developmental Stages}

Newborn No immunoreactive structures were seen in the pineal organ, but a few serotonin fibers were already found in the habenular and posterior commissural regions.

One day after birth Although serotonin- 


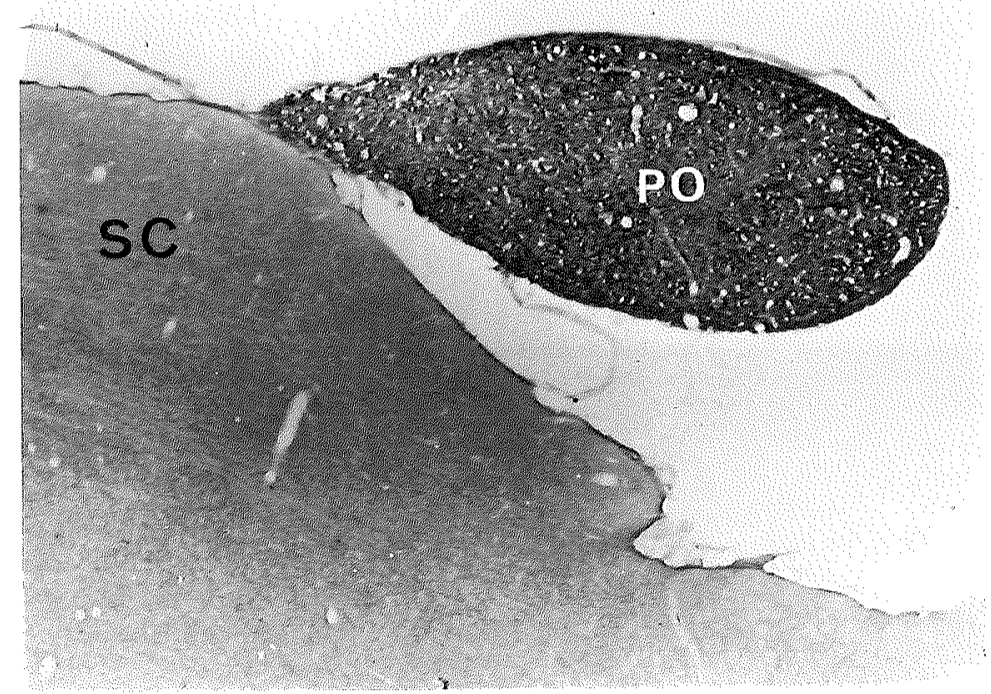

Fig. 1 Paramedian sagittal section through the pineal organ (PO) of the rat. The pineal organ shows intense immunoreactivity. Compare with the superior colliculus (SC). $\times 37$

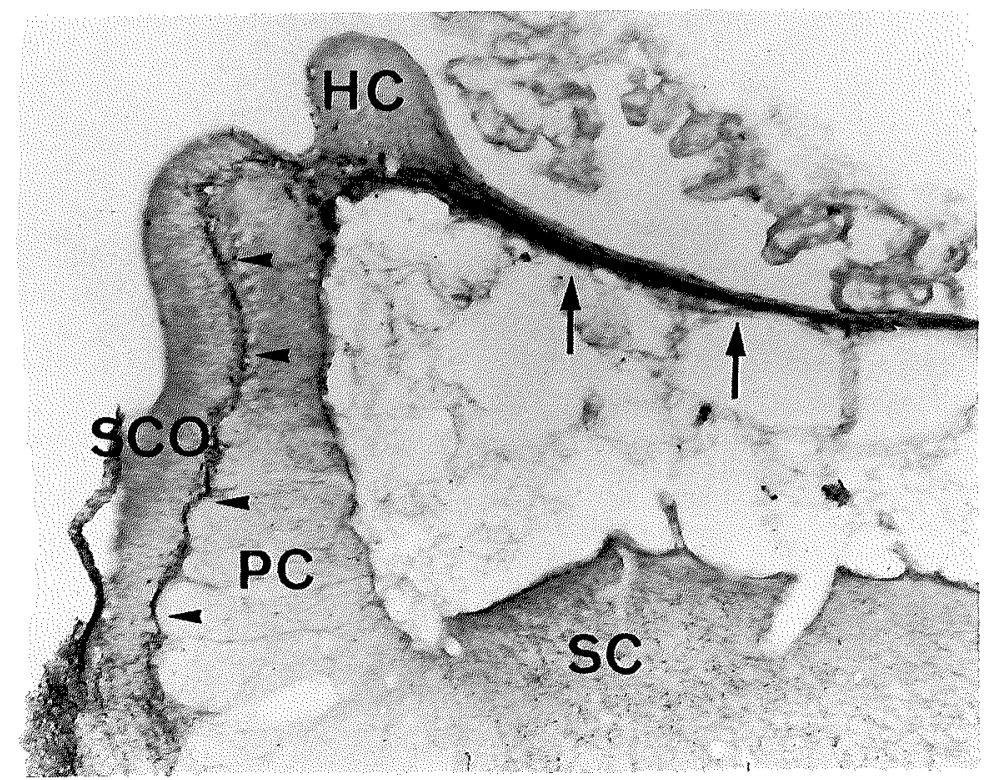

Fig. 2 Sagittal section through the pineal stalk (arrows). Well-developed serotonergic fibers (arrowheads) are observed in the contact zone between the subcommissural organ (SCO) and the posterior commissure (PC) as well as on the surface of the habenular commissure (HC). Some of these serotonergic fibers enter the pineal stalk. The network of serotonergic fibers in the superior colliculus (SC) is clearly shown. The serotonergic supraependymal plexus is also shown at bottom left. $\times 98$ 

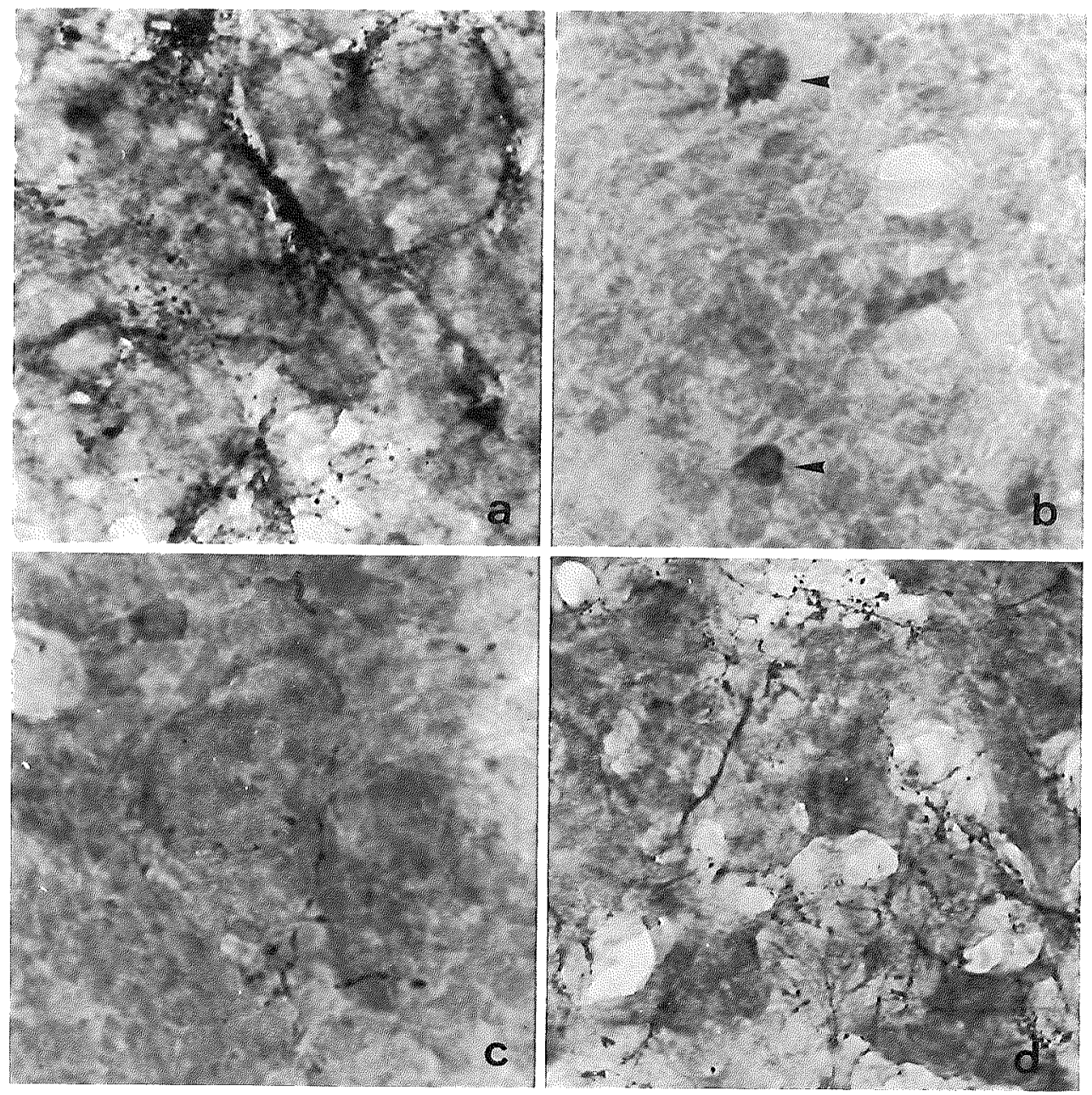

Fig. 3 Immunohistochemical features in the pineal organ at various development stages.

a: Adult. Many immunoreactive varicose fibers are distributed along the blood vessels. Some of them penetrate into the intercellular space of the parenchymal cells. $\times 600$

b: One day after birth. Although a few immunopositive pinealocytes (arrowheads) are scattered in the parenchyma, no immunoreactive nerve fibers are seen at this stage, $\times 700$

c: Three days after birth. Some serotonin nerve fibers have appeared at this stage. They are beaded fibers with varicosities and the intervaricose segment is clearly observed. $\times 740$

$\mathrm{d}$ : Seven days after birth. The distribution pattern of nerve fibers resembles that of the adult. Although the development of varicosities is conspicuous, the number of the nerve fibers is not as large as in the adult and many of them are still beaded fibers, $\times 450$

containing nerve fibers could not yet be detected, a small number of pinealocytes showing positive immunoreaction were scattered in the gland (Fig. 3b).

Three days after birth A few branched serotonin fibers with a small number of varicosities appeared for the first time in the peripheral part of the gland (Fig. 3c). These fibers were distributed along the blood vessels. In this period the varicosities $(1.0 \times 1.5 \mu \mathrm{m})$ were mostly oval, and these as well as the intervaricose segments showed intense reactivity. Immunoreactive parenchymal cells were more numerous than in the animals one day after birth, but many other parenchymal cells still showed no reaction.

Seven days after birth Well-developed serotonin fibers with numerous varicosities were observed throughout the pineal organ (Fig. 3d). 

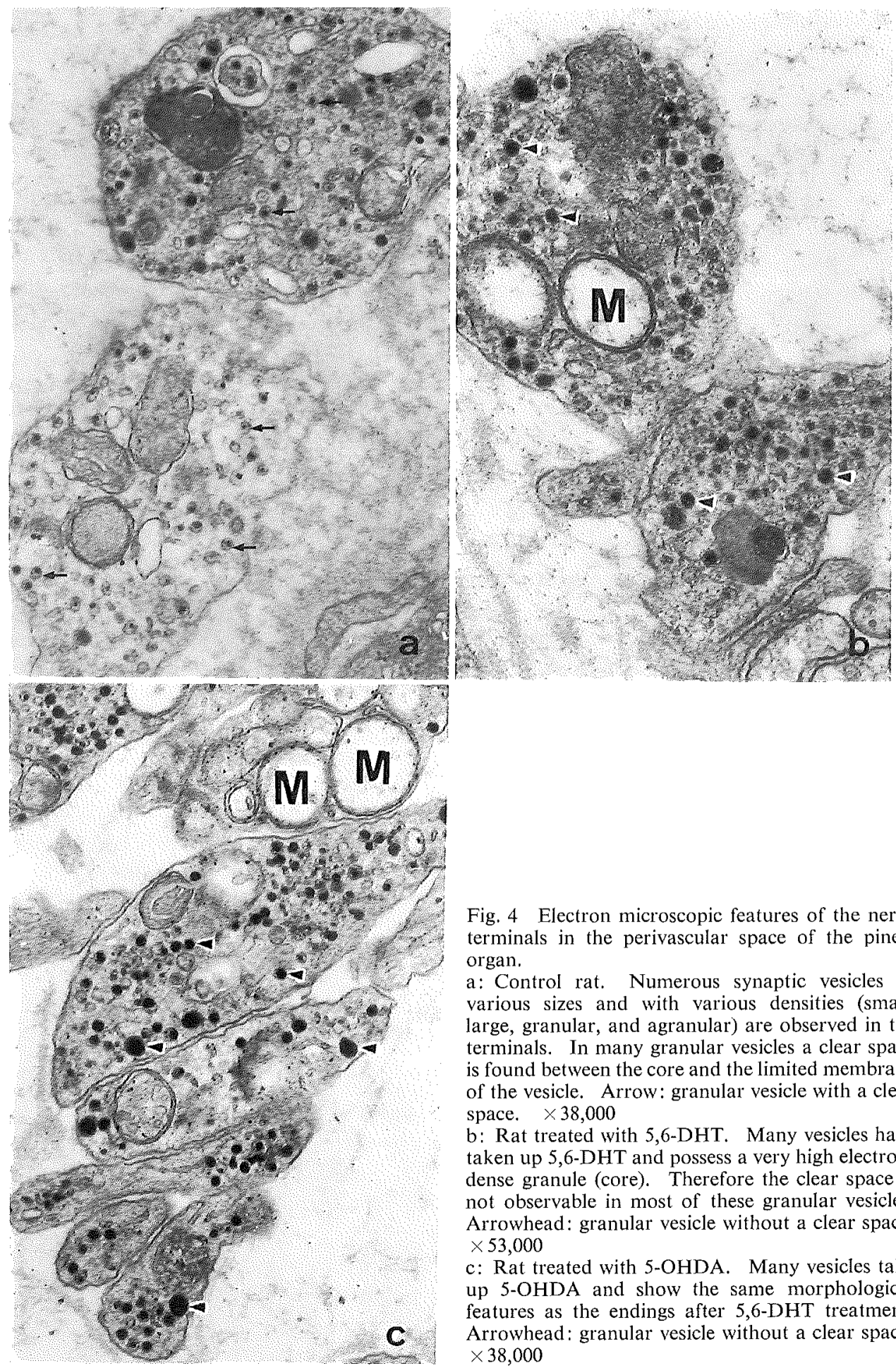

Fig. 4 Electron microscopic features of the nerve terminals in the perivascular space of the pineal organ.

a: Control rat. Numerous synaptic vesicles of various sizes and with various densities (small, large, granular, and agranular) are observed in the terminals. In many granular vesicles a clear space is found between the core and the limited membrane of the vesicle. Arrow: granular vesicle with a clear space. $\times 38,000$

b: Rat treated with 5,6-DHT. Many vesicles have taken up 5,6-DHT and possess a very high electrondense granule (core). Therefore the clear space is not observable in most of these granular vesicles. Arrowhead: granular vesicle without a clear space. $\times 53,000$

c: Rat treated with 5-OHDA. Many vesicles take up 5-OHDA and show the same morphological features as the endings after 5,6-DHT treatment. Arrowhead: granular vesicle without a clear space. $\times 38,000$ 
Intervaricose segments showed rather weak reaction, but the reactivity of varicosities was prominent. All parenchymal cells were strongly stained by the PAP method.

Ten, 14, and 21 days after birth Varicose setotonin fibers further increased in number as days passed. These fibers were not localized in the perivascular region, but distributed in the intercellular space of the parenchymal cells. Formation of varicosities was most prominent at 14 days after birth and their distribution pattern resembled that of the adult animals. Dense networks of varicose fibers were observed in the perivascular spaces and some of them penetrated to the intercellular space of the parenchymal cells. At 21 days after birth, increased ramification of the varicose fibers and increased immunoreactivity could be seen. At this age the morphological features of the pineal organ were the same as those of adult animals.

\section{Submicroscopical Findings}

In the adult rats many small granular and agranular vesicles (40-60 $\mathrm{nm}$ in diameter) and a few large granular and agranular vesicles (90$150 \mathrm{~nm}$ in diameter) were contained in the nerve fibers distributed mainly in the perivascular space of the pineal organ (Fig. 4a). The granular vesicles possessed an eccentrically or centrally located dense core. In most cases a clear space was found between the core and the limiting membrane of the vesicle.

We investigated the quantitative and qualitative changes in the synaptic vesicles in the perivascular nerve fibers ontogenetically. The number of synaptic vesicles was increased with the passage of time after birth, and reached the adult level three weeks after birth. A significant phenomenon was the change in the ratio between granular and agranular vesicles. The number of granular vesicles reached a peak on the 7 th day after birth, when it was $69 \%$ of the total number of synaptic vesicles. The percentage of granular vesicles was gradually decreased after this period, and three weeks after birth it was about $50 \%$ (Table 1 ).

In the animals treated with a false transmitter, viz., 5-OHDA or 5,6-DHT, synaptic vesicles, especially small and large granular vesicles, showed some clear morphological changes. The number of granular vesicles and the electron density of their cores were increased. The space between the core and the limiting membrane disappeared in vesicles that took up the false transmitter. As a result, the ratio of granular vesicles to the total number of synaptic vesicles reached more than $65 \%$ (Table 1); this value was about $15 \%$ higher than in the control animals. The contrast between granular and agranular vesicles was also intensified by the enhanced electron density of the cores. Since the results obtained after 5-OHDA treatment and after 5,6-DHT treatment were very similar, it was difficult to detect any difference in the influence of these transmitters (Fig. 4, b and c).

\section{DISCUSSION}

In the present study the quantitative changes in the serotonin-containing fibers during postnatal development in the rat pineal organ were investigated for the first time by a modified PAP method. Machado et al. (18) and Matsuura (19) demonstrated with the FIFH technique that serotonin fluorescence first appeared on the

Table 1 Number of Total Synaptic Vesicles and Granular Vesicles in the Perivascular Nerve Fibers, and Their Ratio in Normal and Experimental Animals

\begin{tabular}{ccccccccc}
\hline & & & & & & & $\begin{array}{c}\text { Animals } \\
\text { treated } \\
\text { with } \\
\text { 5-OHDA }\end{array}$ & $\begin{array}{c}\text { Animals } \\
\text { treated } \\
\text { with } \\
5 \text { 5-6-DHT }\end{array}$ \\
\hline Day after birth & 3 & 7 & 10 & 14 & 21 & Adult & Adult & Adult \\
\hline $\begin{array}{c}\text { Number of synaptic } \\
\text { vesicles }\end{array}$ & 16 & 45 & 53 & 59 & 75 & 77 & 80 & 77 \\
$\begin{array}{c}\text { Number of granulated } \\
\text { vesicles }\end{array}$ & 3 & 31 & 32 & 32 & 38 & 37 & 52 & 51 \\
$\begin{array}{c}\text { Granulated vesicles } / \\
\text { Synaptic vesicles }(\%)\end{array}$ & 19 & 69 & 60 & 54 & 51 & 48 & 65 & 66 \\
\hline
\end{tabular}

The average number of vesicles per terminal determined with $60-70$ randomly selected terminals from three animals is given. 
third day after birth in the pinealocytes, and on the 6 th or 7 th day after birth in the perivascular nerve fibers. In the present immunohistochemical study positive reaction for serotonin was observed on the first day in the pinealocytes, and on the third day in the nerve fibers, which is earlier than with the FIFH method. The difference between the data obtained by FIFH and the PAP methods may be due to the difference in the sensitivity of both methods; small amounts of serotonin undetectable by FIFH technique may be demonstrable by the PAP method.

We suspect that the 7 th day after birth is a significant point for the process of maturation in the rat pineal organ. At this stage, the immunoreactive parenchymal cells and nerve fibers have increased remarkably in number and the serotonin fibers have expanded to all parts of this organ. These immunohistochemical results are in accord with the results obtained with FIFH by Machado et al. (18) and Matsuura (19). At this time a conspicuous increase in the number of the granular vesicles in the axoplasm of the terminal parts of the pineal nerves was also recognized by electron microscopy.

When observed by the PAP method, the boundary of each parenchymal cell, which was clear in the early stages after birth, became obscure later, especially in mature animals. In the results obtained by the FIFH technique, parenchymal cells of the adult rat pineal organ showed a rather intense yellow fluorescence, but the boundary of each cell was not clear $(19,20)$. It is assumed that these results are due to the following reason. Serotonin richly contained in the parenchymal cells, especially in adult animals, is easily released from the cytoplasm into the intercellular space under physiological conditions, and during the procedure for fixation or histochemical reaction. Serotonin thus released into the intercellular space and detectable by both the FIFH and PAP methods may obscure the cell boundaries. In addition, these cells may overlap in the thick tissue sections.

The serotonin immunoreactivity in the nerve fibers in the pineal organ disappeared completely after bilateral superior cervical ganglionectomy. This suggests that the serotonin-containing fibers in the rat pineal organ are sympathetic postganglionic fibers originating from the superior cervical ganglia. Eränkö and Eränkö using fluorescence histochemistry (12), and Verhofstad et al. using immunohistochemistry (29), observed serotonin-containing small ganglionic cells in the superior cervical ganglion of the rat. However, it is difficult to confirm that these cells extend their processes to the pineal organ, because these ganglionic cells belong to a type of small intensely fluorescent cells (SIF cells) which have been interpreted as interneurons, endocrine cells, or chemoreceptor cells $(9,12$, 17, 30, 31).

There are many studies concerning the effects of the false transmitters, 5-OHDA and 5,6DHT, on the submicroscopical structure of monoamine neurons. According to these investigations, 5-OHDA and 5,6-DHT were respectively taken up by catecholaminergic or indolaminergic terminals $(3,5-7,10,14,24,28)$. In the present study, however, no differences were observed in the effects of these two drugs on the fine structures of the terminals, especially the synaptic vesicles, of the pineal nerve. After treatment with either 5-OHDA or 5,6-DHT, the electron density of the core of the vesicle and the number of granular vesicles were remarkably increased, and the ratio of the granular vesicles constantly showed a value about $15 \%$ higher than in control animals. These results suggest that not only granular vesicles but also agranular vesicles take up 5-OHDA and 5,6-DHT. Duffy and Markesbery (11) have concluded that the storage site of serotonin in noradrenergic fibers of the pineal organ of the rat is granular vesicles, by electron microscopy following treatment with some agents, e.g., reserpine, $p$-chlorphenylalanine and oxypertine. Bak et al. (2) reported, using tritiated 5-HTP, that the serotonin storage site in pineal nerve fibers was both the agranular and granular vesicles in the nerve terminals. On the other hand, Hess (13) suggested that serotonin and noradrenaline were stored only in the granular vesicles. However, it was also demonstrated that these two amines did not exist in the same vesicles when studied electron-microscopically after the administration of reserpine and $p$-chlorphenylalanine to rats and guinea pigs. It was thought that false transmitters for monoamine, such as 5-OHDA, 6-OHDA and 5,6-DHT, increased the osmiophilia of vesicles after being accumulated in the synaptic vesicles of the monoamine nerve terminals, replacing endogenous monoamine $(1,3,4,14-16$, $21,27)$. On the basis of these considerations, our results suggest that serotonin as well as noradrenaline are contained in the same synaptic vesicles in the pineal nerve terminals and that they are enclosed in both granular and agranular vesicles. The idea is supported by the assumption of Duffy and Markesbery (11) that electron 
density of the granular vesicles in the pineal nerve terminals is not entirely due to noradrenaline or serotonin but is related to other substances, such as a binding or carrier substance, and also by the electron microscopical autoradiography by Bak et al. (2).

We have confirmed that the noradrenergic pineal nerve possesses the ability to take up and store serotonin released from the pinealocytes. We speculate that serotonin, after being taken up, is concentrated and stored in a stabilized state, not only in granular vesicles but also in agranular vesicles in the pineal nerve terminals. This ability seems to develop in young rats at a rate corresponding to their growth and maturation.

We wish to thank Professor H. Masuda and Mr D. Raycroft for their advice on language. This work was supported by a grant (No.57214028) from the Ministry of Education, Science and Culture, Japan.

Received for publication 7 February 1983; and in revised form 8 April 1983

\section{REFERENCES}

1. AJIKA K. and HöKfELT T. (1973) Ultrastructural identification of catecholamine neurons in the hypothalamic periventricular-arcuate nucleusmedian eminence complex with special reference to quantitative aspects. Brain Res. 57, 97-117

2. BAK I. J., KIM J. H. and HASSLER R. (1970) Electron microscopic autoradiography for demonstration of pineal serotonin in rat. $Z$. Zellforsch. 105, 167-175

3. Baumgarten H. G., Buörklund A., Holstein A. F. and Nobin A. (1972) Chemical degeneration of indolamine axons in rat brain by 5,6 dihydroxytryptamine. An ultrastructural study. Z. Zellforsch. 129, 256-271

4. Bennett T., Burnstock G., СobB J. L. S. and Malmfors T. (1970) An ultrastructural and histochemical study of the short-term effects of 6-hydroxydopamine on adrenergic nerves in the domestic fowl. Brit. J. Pharmacol. 38, 802-809

5. Bertler Å., Falck B. and Owman Ch. (1964) Studies on 5-hydroxytryptamine stores in pineal gland of rat. Acta Physiol. Scand. 63, Suppl. $239,1-18$

6. Björklund A., Baumgarten H. G. and Nobin A. (1974) Chemical lesioning of central monoamine axons by means of 5,6-dihydroxytryptamine and 5,7-dihydroxytryptamine. In Serotonin: New Vistas Histochemistry and Pharmacology (ed. Costa E., Gessa G. L. and SAndler M.) Advances in Biochemical Psycopharmacology Vol. 10, Raven Press, New York, pp. 13-33

7. Buörklund A., Nobin A. and Stenevi U. (1973) Effects of 5,6-dihydroxytryptamine on nerve terminal serotonin and serotonin uptake in the rat brain. Brain Res. 53, 117-127

8. Buörklund A., OWman $\mathrm{CH}$, and West K. A (1972) Peripheral sympathetic innervation and serotonin cells in the habenular region of the rat brain. Z. Zellforsch. 127, 570-579

9. CHIBA T. (1977) Monoamine-containing paraneurons in the sympathetic ganglia of mammals. Arch. histol. japon. 40, Suppl., 163-176

10. Daly J., Fuxe K. and Jonsson G. (1973) Effects of intracerebral injections of 5,6-dihydroxytryptamine on central monoamine neurons: Evidence for selective degeneration of central 5-hydroxytryptamine neurons. Brain Res. 49, 476-482

11. Duffy P. E. and Markesbery W. R. (1970) Glanulated vesicles in sympathetic nerve endings in the pineal gland: Observations on the effects of pharmacologic agents by electron microscopy. Amer. J. Anat. 128, 97-116

12. ERÄNKö O. and ERÄNKÖ L. (1971) Small, intensely fluorescent granule-containing cells in the sympathetic ganglion of the rat. Prog. Brain Res. 34, 39-51

13. Hess H. (1981) Localization of noradrenaline and serotonin in nerves in the pineal gland of rats and guinea pigs studied by glyoxylic acid histofluorescence and electron microscopy. Histochem. J. 13, 425-434

14. Ibata Y., Matsuura T., Nojyo Y., Inoue $T$ and SANO Y. (1974) The effect of 5- and 6hydroxydopamine on the central monoaminergic neurons of the rat and the cat: Fluorescence histochemistry and electron microscopy. Acta Histochem. Cytochem. 7, 126-139

15. Ibata Y., Nojyo Y., Matsuura T. and Sano Y. (1974) The effect of high dosage of 5-hydroxydopamine on the arcuate nucleus of the cat and its possible uptake by the catecholamine-containing neuronal soma, J. Electr. Micr. 23, 295-299

16. Ibata Y., Nojyo Y., Matsuura T., Yoshikawa H. and Sano Y. (1975) Electron microscopy of the arcuate nucleus of normal and 5-hydroxydopamine treated cats. Cell Tissue Res. 160, 139-153

17. Lu K.-S., Lever J. D., SAnter R. M. and Presley R. (1976) Small granulated cell types in rat superior cervical and coeliac-mesenteric ganglia. Cell Tissue Res, 172, 331-343

18. Machado C.R.S., Wragg L. E. and Machado A. B. M. (1968) A histochemical study of sympathetic innervation and 5-hydroxytryptamine in the developing pineal body of the rat. Brain Res. 8, 310-318

19. Matsuura T. (1972) Ontogenesis of autonomic nerve fibers distributing in the pineal body of the rat. Aut. Nerv. Syst. 9, 229-236 (in Japanese)

20. Matsuura T. (1977) The influence of chemical and surgical sympathectomy on the rat pineal body. J. Kyoto Pref. Univ. Med. 86, 493-507

21. Mazzuca M. et Poulain P. (1974) Identification en microscopie électronique des terminaisons nerveuses monoaminergiques dans l'éminence 
médiane du cobaye. Brain Res. 68, 281-295

22. OWman CH. (1964) New aspects of the mammalian pineal gland. Acta Physiol. Scand. 63, Suppl. 240, 1-40

23. OWMAN CH. (1965) Localization of neuronal and parenchymal monoamines under normal and experimental conditions in the mammalian pineal gland. Prog. Brain Res. 10, 423-453

24. Richards J. G. and Tranzer J.-P. (1970) The ultrastructural localisation of amine storage sites in the central nervous system with the aid of a specific marker, 5-hydroxydopamine. Brain Res. 17, 463-469

25. Takeuchi Y, Kimura H. and Sano Y. (1982) Immunohistochemical demonstration of the distribution of serotonin neurons in the brainstem of the rat and cat. Cell Tissue Res. 224, 247-267

26. Tilders F. J. H., Ploem J. S. and Smelik P. G. (1974) Quantitative microfluorimetric studies on formaldehyde-induced fluorescence of 5-hydroxytryptamine in the pineal gland of the rat. $J$. Histochem. Cytochem. 22, 967-975

27. Tranzer J. P. and Richards J. G. (1971) Fine structural aspects of the effect of 6-hydroxydopamine on peripheral adrenergic neurons. In 6-Hydroxydopamine and Catecholamine Neurons (ed. Malmfors T. and Thoenen H.) NorthHolland, Amsterdam, pp. 15-31
28. Tranzer J, P. and Thoenen H. (1967) Electronmicroscopic localization of 5-hydroxydopamine (3,4,5-trihydroxyphenyl-ethylamine), a new 'false' sympathetic transmitter. Experientia (Basel) 23, 743-745

29. Verhofstad A. A. J., Steinbusch H. W. M., Penke B., Varga J. and Joosten H. W. J. (1981) Serotonin-immunoreactive cells in the superior cervical ganglion of the rat. Evidence for the existence of separate serotonin- and catecholamine-containing small ganglionic cells. Brain Res. 212, 39-49

30. Williams T. H., Black A. C., Chiba T. and JEW J. (1976) Interneurons/SIF cells in sympathetic ganglia of various mammals. In Chromaffin, Enterochromaffin and Related Cells. (ed. Coupland R. E. and Fujita T.) Elsevier, Amsterdam, pp. 96-116

31. Williams T. H., Chiba T., Black A. C., Bhalla R. C. and JEW J. (1976) Species variation in SIF cells of superior cervical ganglia: Are there two functional types? In SIF Cells. Structure and Function of the Small Intensely Fluorescent Sympathetic Cells (ed. ERÄNKö O.) Forgary International Center Proceedings No. 30, U.S. Government Printing Office, Washington D.C., pp. 143 162 